\title{
Exocrine pancreatic insufficiency and causes of abdominal symptoms in diabetes patients
}

\author{
Roland Kadaj-Lipka ${ }^{1}$, Grażyna Rydzewska ${ }^{1,2}$ \\ ${ }^{1}$ Clinical Department of Internal Medicine and Gastroenterology with Inflammatory Bowel Disease Unit, Central Clinical \\ Hospital of the MSWiA, Warsaw, Poland \\ ${ }^{2}$ Faculty of Medicine and Health Sciences, Jan Kochanowski University, Kielce, Poland
}

Gastroenterology Rev 2020; 15 (4): 289-293

DOI: https://doi.org/10.5114/pg.2020.101556

Key words: diabetes mellitus, pancreatic exocrine insufficiency, small intestinal bacterial overgrowth, gastroparesis, neuropathy.

Address for correspondence: Roland Kadaj-Lipka, Clinical Department of Internal Medicine and Gastroenterology with Inflammatory Bowel Disease Unit, Central Clinical Hospital of the MSWiA, Warsaw, Poland, phone: +48 22508 12 40, e-mail: rolandkadaj@gmail.com

\begin{abstract}
The pancreas has dual functions, endocrine and exocrine, that complement each other and work closely together, contributing to the digestion, absorption, and metabolism of foods. More than a decade ago, it was demonstrated that pancreatic exocrine insufficiency occurs in a significant number of patients with diabetes mellitus. Diabetes mellitus affects virtually every organ system in the body, and the degree of organ involvement depends on the duration and severity of the disease, as well as other co-morbidities. Gastrointestinal side effects include pancreatopathy, small intestinal bacterial overgrowth, oesophageal dysmotility, gastroesophageal reflux disease, gastroparesis, neuropathy, and nonalcoholic fatty liver disease.
\end{abstract}

\section{Introduction}

Diabetes is a disease affecting the whole body. The impact on individual organs depends on the duration of diabetes, its severity, and concomitant diseases [1]. As for the digestive system, complications are most likely to affect the pancreas, stomach, oesophagus, and intestines. Abdominal symptoms in patients with diabetes are becoming increasingly common because the number of patients with diabetes is growing steadily $[2,3]$. About $75 \%$ of patients treated in diabetic outpatient clinics report persistent gastrointestinal symptoms (GI) [4]. The most frequently reported GI problems in those patients include constipation, indigestion, abdominal pain, and gastroesophageal reflux [5].

In this article, we discuss the pathology and diagnosis of pancreatopathy and exocrine pancreatic insufficiency in diabetic patients. Also, we present the most common causes of abdominal symptoms in diabetes, including small intestinal bacterial overgrowth (SIBO), gastroparesis, diabetic neuropathy, gastroesophageal reflux disease, and pancreatic cancer.

\section{Exocrine pancreatic insufficiency in diabetes patients}

The pancreas has dual endocrine and exocrine functions, complementary to each other for the digestion, absorption, and metabolism of foods. More than a decade ago it was demonstrated that pancreatic exocrine insufficiency (PEI) occurs in a significant proportion of diabetic patients [6]. Pancreatic dysfunction has been proven not only in approximately $50 \%$ of patients with insulin-dependent diabetes, but also in $30-50 \%$ of patients with non-insulin-dependent diabetes mellitus. Also, there have been many studies confirming abnormal pancreatic morphology and histology in such patients [7]. Similarly, damage to pancreatic islets leads to secretory dysfunction and development of a specific type of diabetes (type 3) [8].

There has been little research on PEI in diabetic patients, and most studies were carried out on small patient groups, in which pancreatic dysfunction was interpreted as a complication of diabetes. Only a few hypotheses point to exocrine pancreatic function as diabetes-induced pancreatopathy [6]. Due to a lack of 
unambiguous results and conclusions, no guidelines or therapeutic recommendations have been developed for diabetic patients with pancreatic exocrine insufficiency.

Type 1 diabetes is driven by a primary autoimmune process and is characterised by early occurrence, severe insulin deficiency, long duration, and high incidence of neurological and vascular complications. It seems to be more often associated with PEI than with type 2 . As for type 2 diabetes, the following risk factors for exocrine insufficiency have been suggested: early disease onset, long duration, and poor glucose control (expressed specifically in terms of elevated glycated haemoglobin $\left(\mathrm{HbA}_{1 \mathrm{c}}\right)$ level). Nevertheless, the impact of those factors was not statistically significant in every study. This supports the hypothesis that long-lasting complicated type 2 diabetes is associated with a higher degree of microcirculatory damage, pancreatic fibrosis, and neuropathy, and probably development of PEI [8].

In the largest prospective study conducted on a group of 1231 diabetic patients, Hardt et al. observed PEI in 697 (35\%) patients with type 2 diabetes. It is worth noting that the study did not exclude patients with previous history of pancreatic disease, which may have skewed the results [8].

Other studies, involving smaller numbers of patients, confirmed the incidence of PEI in $30-40 \%$ of patients with type 2 diabetes. Most studies also show that the need for insulin therapy is associated with a higher incidence and a more severe course of PEI [8].

Pancreatic insufficiency was more frequent in diabetic patients (36\%) than in the control group (5\%) [9]. Diabetic or overweight (body mass index (BMI) over $25 \mathrm{~kg} / \mathrm{m}^{2}$ ) patients have increased risk of exocrine pancreatic insufficiency $[8,9]$.

The knowledge about symptoms of pancreatic exocrine insufficiency in diabetic patients is low. Some symptoms, such as abdominal pain, weight loss, nausea, and diarrhoea, can also be attributed to other gastrointestinal diseases such as irritable bowel syndrome (IBS), peptic ulcer disease, SIBO, and so on. Therefore, many patients with $\mathrm{PEI}$ are not correctly diagnosed and treated [10].

\section{Diagnosis of pancreatic exocrine insufficiency}

Several types of diagnostic tests are used to assess pancreatic exocrine function, including direct and indirect tests. The advantage of functional tests is that they allow the identification and evaluation of pancreatic exocrine insufficiency at its early stages before advanced damage develops, signified by obvious symptoms such as parenchymal calcifications or fatty stools.
The evaluation of elastase 1 activity in stool is an example of an indirect test. Pancreatic elastase 1 is a very specific human protease (proteolytic enzyme) secreted by pancreatic cells within acini. Elastase 1 is a very stable enzyme, which is not degraded in the alimentary tract, unlike other pancreatic proteases (e.g. chymotrypsin), reflecting pancreatic secretory function; for this reason, it is a good diagnostic test [8]. Indirect testing for faecal elastase 1 applies monoclonal antibody detection (ELISA). This test shows high sensitivity, and its advantages include relatively low cost and non-invasive character [11]. Faecal elastase 1 activity over $200 \mu \mathrm{g} / \mathrm{g}$ is considered normal, reflecting normal pancreatic exocrine function, while the activity within the range of $100-200 \mu \mathrm{g} / \mathrm{g}$ is indicative of a slight pancreatic exocrine insufficiency, and below 100 mg/g suggests severe insufficiency [12].

Another diagnostic test evaluating pancreas function is serum lipase activity, which is an enzyme responsible for hydrolysis of triglycerides to di- and monoglycerides and fatty acids.

Endoscopic ultrasound (EUS) is considered one of the most accurate diagnostic methods in pancreatic disease. It is used for morphological evaluation of the pancreas, which in combination with indirect tests allows the comprehensive evaluation of the exocrine function. Endoscopy may also provide treatment for patients with pancreatic disorders, when medical therapy has proven ineffective [13].

\section{Causes of abdominal symptoms in diabetic patients}

Diabetic neuropathy and small intestinal bacterial overgrowth

Functional disorders of the small and large intestine are relatively frequent in patients with long-lasting diabetes, especially complicated by gastroparesis [1, 4]. Diabetic neuropathy may present with symptoms such as diarrhoea, constipation, or faecal incontinence. The pathological basis for enteropathy is similar to that responsible for upper Gl symptoms. Advanced glycation end-products (AGEs) result from prolonged elevation of serum glucose level, causing damage to cellular DNA and tissues. Neuronal damage and fibrosis of the intestinal muscular layer lead to stasis [1]. Reduced intestinal motility promotes constipation, which in turn may cause incontinence in some cases. As a consequence of bowel content retention, small intestinal bacterial overgrowth syndrome (SIBO) can ensue. Constipation alternating with diarrhoea is typical for diabetic neuropathy. Diarrhoea is usually painful; it may occur during the day, but usually it occurs at night $[14,15]$. The most common symptoms can be observed in patients with poorly controlled diabe- 
tes, who show symptoms of peripheral and autonomic neuropathy [16]. Other causes of diabetes-associated diarrhoea include pancreatic exocrine insufficiency or metformin use [1]. Those causes should be excluded before the diagnosis of diabetic neuropathy is made.

Constipation is a problem for almost $60 \%$ of patients with long-lasting diabetes. Chronic hyperglycaemia impairs physiological function of the anal sphincter. In such cases, endoscopy and diagnostic work-up for SIBO are recommended, which can often accompany intestinal content retention.

Small intestinal bacterial overgrowth is an abnormal growth of the large intestinal bacteria within the small intestine. Bacterial overgrowth in the intestines can cause problems such as abdominal pain, bloating, diarrhoea, and constipation. SIBO can be diagnosed using a variety of methods, but because of its low cost and non-invasive character, the hydrogen breath test is the most widely available study [17]. It is based on hydrogen detection, which forms in the intestines as a result of carbohydrate fermentation by bacteria. Through circulation, the hydrogen passes into the alveoli and is excreted by the respiratory system. The human body does not produce hydrogen when there is a physiological balance of intestinal bacteria, and thus its presence clearly indicates bacterial overgrowth.

Treatment of diarrhoea caused by diabetic neuropathy is symptomatic, focusing on maintaining water and electrolyte balance and normoglycaemia [1]. Chronic use of anti-diarrhoeal medication is not recommended. Use of rifaximin is recommended. It is a non-absorbable antibiotic, administered orally, with a broad spectrum of activity including Gram-positive, Gram-negative, aerobic, and anaerobic bacteria, and it is recommended in SIBO [18]. After a rifaximin course, patients show $84 \%$ reduction in non-physiological bacterial flora [1].

\section{Gastroparesis}

Gastroparesis is one of the most common complications of diabetes, causing abnormal gastric emptying in the absence of physical obstruction. The incidence of gastroparesis in the diabetic population is $5.2 \%$ per 10 years for type 1 and 1\% for type 2 diabetes, but it is definitely higher compared to the general population $(0.2 \%)$. The incidence of this complication is higher in women. Based on a recent study, obesity was suggested to be a significant independent risk factor for gastroparesis in patients with type 2 diabetes and neuropathy [1].

The pathogenesis of gastroparesis is multifactorial and not fully understood [1]. Delayed gastric emptying is the first symptom of diabetic gastroparesis. Other symptoms include nausea, vomiting, early satiety, flatulence, and abdominal pain. About $53 \%$ of patients report weight loss, and a further $18-24 \%$ report gain weight [1].

Diagnosis of gastroparesis, according to the American Gastroenterological Association (AGA) guidelines, is based on a detailed patient history, physical examination, and blood tests (such as thyroid hormones and amylase); gastroscopy is indicated to to rule out mechanical obstruction [1]. In abdominal pain, ultrasound is also recommended to assess the gallbladder for potential gallstones. The diagnosis of gastroparesis can also be confirmed by gastric scintigraphy, which allows an evaluation of the passage of food and track stomach emptying [1, 3].

Treatment of gastroparesis consists of modification of eating habits, pharmacotherapy, weakening of gag reflex, psychological care, or surgical treatment. General nutritional recommendations for delayed gastric emptying focus on maintaining water and electrolyte balance and normoglycaemia, promoting liquid meals, reducing fat and hard-to-digest fibre intake, reducing energy-rich meals, and preferring many smaller meals during the day [1].

The recommended pharmacotherapy for gastroparesis includes prokinetics, which enhance stomach motility and accelerate emptying. Other medications include the following: Metoclopramide - it shows antiemetic activity, reduces nausea and postprandial early satiety, and increases gastroesophageal sphincter tone; Ondansetron it is a selective antagonist of 5-HT3 serotonin receptors in the CNS and in the vagal nerve endings; Domperidone - it has an effect similar to metoclopramide, but shows less CNS side effects; Erythromycin may also be used in therapy to shorten gastric emptying time.

Mearin et al. point out pyloric contraction as a factor delaying gastric emptying $[1,14]$. Endoscopic injection of botulinum toxin causes pyloric paralysis, which resulted in faster gastric emptying for 3-6 months in women with idiopathic gastroparesis as well as in older men with vomiting. However, surgical intervention is rarely indicated, and it is mainly used for people with persistent symptoms despite adequate medical treatment [1].

\section{Gastroesophageal reflux disease}

Some studies indicate that gastroesophageal reflux disease (GERD) is more common in diabetic patients than in non-diabetics [19-21].

Based on endoscopic studies, up to $42 \%$ of type 2 diabetes patients develop gastroesophageal reflux disease [21]. In those studies, patients with concurrent diabetes and gastroesophageal reflux disease tended to be younger, with a shorter duration of diabetes, were taller, and had greater body mass. There was also a significant relationship between GERD and gender, showing that 
the complicated form of GERD was more frequent in men. However, there are no significant associations between the presence of reflux disease and other diabetic complications [21].

Gastroesophageal reflux disease not only affects the patients' quality of life, but also increases the risk of oesophageal adenocarcinoma [22]. The main pathomechanism of GERD is a transient relaxation of the lower oesophageal sphincter. Research has shown that overweight and obesity are important risk factors for gastroesophageal reflux disease. One of the mechanisms consists of obesity leading to an increased gradient across the gastroesophageal sphincter, predisposing for hiatus hernia and increased intrabdominal pressure [22] Some diabetic patients, especially those with type 2 diabetes, are obese, this suggests why diabetes patients may have GERD [22].

\section{Pancreatic cancer risk in diabetes patients}

Long-lasting diabetes causes a twofold increase in the risk for pancreatic cancer. Therefore, this group of patients requires special attention considering pancreatic diseases. As far as pancreatic cancer patients are concerned, $50 \%$ of them develop diabetes, which indicates a correlation between diabetes and pancreatic cancer [20].

The most common and fatal type of pancreatic cancer is pancreatic adenocarcinoma (PDAC), which is closely associated with diabetes mellitus. Among 100 patients diagnosed with lung, breast, colon, prostate, or PDAC, $68 \%$ of PDAC patients had concurrent diabetes, whereas the incidence of diabetes in other cancers was 15 to $21 \%$ [23]. Long-lasting type 2 diabetes is a risk factor for pancreatic cancer, but the latest epidemiological data suggest that PDAC can lead to diabetes in a mechanism yet unknown [23]. Importantly, in 74-88\% of patients with pancreatic ductal adenocarcinomas and concurrent diabetes, diabetes developed less than 24 months before the diagnosis of cancer [23]. Those facts suggest that diabetes and pancreatic cancer can show mutual coincidence. Long-lasting type 2 diabetes is a risk factor for developing pancreatic cancer, and, in turn, PDAC can probably cause diabetes. The mechanisms for this mutual relationship are still unclear, and so are the diagnostic criteria for diabetes occurring as an early complication of pancreatic cancer [23].

Pancreatic exocrine insufficiency usually precedes diabetes in patients with a history of acute or chronic pancreatitis [23]. Each episode of acute pancreatitis may trigger transient glucose intolerance and increases the risk of diabetes later in life [23].

The incidence of diabetes among patients with pancreatic cancer is extremely high. In a study including
512 PDAC patients and 933 control volunteers, diabetes was diagnosed in $47 \%$ of cancer patients compared to only $7 \%$ in the control group. In $74 \%$ of patients with pancreatic cancer, the onset of diabetes was within 24 months before the diagnosis of cancer, often at the time when the tumour was already detectable on imaging. It suggests that, in many cases, newly diagnosed diabetes can be precipitated by a pancreatic cancer and may be the first signal warranting oncological workup [23].

\section{A new Polish study relates to abdominal symptoms in diabetic patients with special focus on pancreatic exocrine insufficiency}

The relationship between pancreatopathy and diabetes was established 70 years ago, but the scope of this relationship has not been fully understood until recently. In a large-scale study on diabetic patients, 51.1\% of type 1 and $35.4 \%$ of type 2 diabetes patients showed reduced faecal elastase 1 activity, which is an indirect test for the evaluation of pancreatic exocrine function and gastrointestinal complaints. In $17.8 \%$ of all diabetic patients, mild impairment of exocrine pancreatic function was observed, and another $22.9 \%$ had severe pancreatic dysfunction. The results as well as the correlation between faecal elastase 1 activity and diabetes duration lead to the conclusion that faecal elastase 1 and pancreatic secretory capacity should be regularly monitored in this group of patients [10].

Faecal elastase 1 testing has also been implemented in an ongoing Polish study conducted at the Clinical Department of Internal Medicine and Gastroenterology, CSK MSWiA, Warsaw, aiming to evaluate the causes of abdominal symptoms in diabetic patients with a special focus on pancreatic exocrine dysfunction. It is a prospective, cohort, non-randomised study, including 231 patients with type 1 or 2 diabetes. Seventy-seven patients were excluded from the study because of a previous history of pancreatic diseases, alcohol abuse, or smoking. Sixty-seven patients met the inclusion criteria such as the presence of significant abdominal symptoms, while 87 patients from the study group did not present any significant complaints. Further analysis was conducted on 50 patients; 30 of them presenting abdominal symptoms (test group) and 20 without any symptoms (control group). In all patients, $\mathrm{HbA}_{1 c}$, faecal elastase 1 , lipase, and hydrogen breath test were obtained. In the test group, there was a decrease in elastase 1 concentration below $200 \mu \mathrm{g} / \mathrm{g}$ in $13.33 \%$ of diabetic patients, compared to $15 \%$ in the control group. In contrast, an increased elastase 1 activity above $60 \mathrm{IU} / \mathrm{I}$ was found in $40 \%$ in the test group compared to $15 \%$ in the control group. Hence, we can assume that 
changes in lipase and FE-1 in diabetic patients may reflect development of pancreatopathy in the course of diabetes, or so-called diabetic pancreatopathy, or chronic pancreatitis [20].

In this study the authors also applied diagnostic workup for SIBO including hydrogen breath test (with the test group of 30 patients and the control group of 20). In the test group, SIBO was more frequent in patients with abdominal symptoms (66.67\%) than in the control group (15\%). However, there was no relationship between lipase level or FE-1 and abdominal symptoms such as constipation, diarrhoea, gastroesophageal reflux, dyspepsia, or abdominal pain [19]. The results indicate that abdominal symptoms in diabetic patients are more commonly associated with bacterial overgrowth (SIBO) [19].

\section{Summary}

Managing abdominal symptoms in diabetic patients is difficult because their exact pathomechanism is still unknown. There are few publications and studies on this subject, as well as a lack of guidelines and therapeutic recommendations for this group of patients. Gastrointestinal symptoms, although reported by patients, often remain undiagnosed and ignored, which only hinders further management. In most cases, those symptoms are attributed to diabetic neuropathy by default. In a recent Polish study, preliminary results show that abdominal symptoms in diabetic patients are more commonly associated with bacterial overgrowth (SIBO) [19].

A professional approach to gastrointestinal complications is important to improve quality of life, as well as to provide appropriate monitoring and a high standard of care for diabetic patients.

\section{Conflict of interest}

The authors declare no conflict of interest.

\section{References}

1. Krishnan B, Babu S, Walker J, et al. Gastrointestinal complications of diabetes mellitus. World J Diabetes 2013; 4: 51-63.

2. Economic Costs of Diabetes in the U.S. in 2002, Report from the American Diabetes Association. Diabetes Care 2003; 26: 917-32.

3. Shakil A, Church RJ, Shobhas R. Gastrointestinal complications of diabetes. Am Fam Physician 2008; 77: 1697-702, 1703-4.

4. Zhao JB, Brøndum Frøkjær J, Mohr Drewes A, et al. Upper gastrointestinal sensory-motor dysfunction in diabetes mellitus. World J Gastroenterol 2006; 12: 2846-57.

5. Talley NJ, Young L, Bytzer P, et al. Impact of chronic gastrointestinal symptoms in diabetes mellitus on health-related quality of life. Am J Gastroenterol 2001; 96: 71-6.

6. Hardt DH, Nils E. Exocrine pancreatic insufficiency in diabetes mellitus: a complication of diabetic neuropathy or different type of diabetes? Exp Diabetes Res 2011; 2011; 761950.
7. Hardt PD, Hauenschild A, Nalop J, et al. High prevalence of exocrine pancreatic insufficiency in diabetes mellitus. A multicenter study screening fecal elastase 1 concentrations in 1,021 diabetic patients. Pancreatology 2003; 3: 395-402.

8. Piciucchi M, Capurso G, Archibugi L, et al. Exocrine pancreatic insufficiency in diabetic patients: prevalence, mechanisms, and treatment. Int J Endocrinol 2015; 2015: 595649.

9. Nunes A, Pontes J, Rosa A, et al. Screening for pancreatic exocrine insufficiency in patients with diabetes mellitus. Am J Gastroenterol 2003; 98: 2672-5.

10. Dominguez-Munoz JE, Hardt PD, Lerch MM, et al. Potential for screening for pancreatic exocrine insufficiency using the fecal elastase-1 test. Dig Dis Sci 2017; 62: 1119-30.

11. Dominici R, Franzini C. Fecal elastase-1 as a test for pancreatic function: a review. Clin Chem Lab Med 2002; 40: 325-32.

12. Terzin V, Várkonyi T, Szabolcs A, et al. Prevalence of exocrine pancreatic insufficiency in type 2 diabetes mellitus with poor glycemic control. Pancreatology 2014; 14: 356-60.

13. Magge S, Lembo A. Low-FODMAP diet for treatment of irritable bowel syndrome. Gastroenterol Hepatol 2012; 8: 739-45.

14. Mearin F, Camilleri M, Malagelada JR. Pyloric dysfunction in diabetics with recurrent nausea and vomiting, Gastroenterology 1986; 90: 1919-25.

15. Lee TH, Lee JS. Ramosetron might be useful for treating diabetic diarrhea with a rapid small bowel transit time. Korean J Intern Med 2013; 28: 106-7.

16. Lysy J, Israeli E, Goldin E. The prevalence of chronic diarrhea among diabetic patients. Am J Gastroenterol 1999; 94: 216570.

17. Hong-Yan Q, Chung-Wah C, Xu-Dong T, et al. Impact of psychological stress on irritable bowel syndrome. World J Gastroenterol 2014; 20: 14126-31.

18. Pimentel M, Lembo A, Chey WD. Rifaximin therapy for patients with irritable bowel syndrome without constipation. N Engl J Med 2011; 364: 22-32.

19. Kadaj-Lipka R, Juchnicka A, Rydzewska G. Gastrointestinal symptoms in diabetic patients - diabetic exocrine pancreatopathy or small intestinal bacterial overgrowth? Pancreatology 2017; 17 Suppl. 69.

20. Franek E, Walicka M. Leczenie powikłań cukrzycy i chorób z nią wspótistniejących. Wydawnictwo San Rogue, Warszawa 2018; 235-45.

21. Fujiwara M, Miwa T, Kawai T, et al. Gastroesophageal reflux disease in patients with diabetes: preliminary study. J Gastroenterol Hepatol 2015; 30 (Suppl. 1): 31-5.

22. Sun XM, Tan JC, Zhu Y, Lin L. Association between diabetes mellitus and gastroesophageal reflux disease: a meta-analysis. World J Gastroenterol 2015; 21: 3085-92.

23. Andersen DK, Korc M, Petersen GM, et al. Diabetes, pancreatogenic diabetes, and pancreatic cancer. Diabetes 2017; 66: 1103-10.

Received: 24.10.2019

Accepted: 7.01.2020 